\title{
Protein-losing Gastroenteropathy Related to Mixed Connective Tissue Disease: A Case Report of a Successful Outcome and Literature Review
}

\author{
Yuya Kobayashi, Yasuhiro Shimojima, Yasufumi Kondo, Ryota Takamatsu, Daigo Miyazaki, \\ Dai Kishida, Yoshiki Sekijima and Shu-ichi Ikeda
}

\begin{abstract}
We herein report the case of a 44-year-old woman who developed protein-losing gastroenteropathy (PLGE) with hypoalbuminemia as the first manifestation of mixed connective tissue disease (MCTD). Albumin leakage from the stomach and intestinal tract was demonstrated by ${ }^{99 m} \mathrm{Tc}$-labeled human serum albumin scintigraphy. The patient's response to prednisolone therapy was insufficient; therefore, additional cyclosporin A (CsA) treatment was administered, and clinical remission was achieved. We concluded that although PLGE is a rare complication of MCTD, it may manifest as an initial clinical episode of MCTD. Furthermore, CsA can be a useful treatment option for refractory PLGE related to MCTD.
\end{abstract}

Key words: mixed connective tissue disease, protein-losing gastroenteropathy, hypoalbuminemia, ${ }^{99 \mathrm{~m}} \mathrm{Tc}-$ labeled human serum albumin scintigraphy, cyclosporin A

(Intern Med 56: 2057-2062, 2017)

(DOI: 10.2169/internalmedicine.56.8391)

\section{Introduction}

Protein-losing gastroenteropathy (PLGE) is a disorder characterized by a leakage of serum protein into the gastrointestinal tract, resulting in hypoproteinemia, which in turn leads to general edema, ascites, and pleural and pericardial effusions (1). Various disorders are known to be associated with PLGE; however, it is a rare complication of autoimmune diseases (2). Among these, systemic lupus erythematosus (SLE) has been recognized as a relatively common cause of PLGE, while mixed connective tissue disease (MCTD) is a rare cause $(2,3)$. We herein report a case of PLGE that presented as an initial clinical episode of MCTD. Successful treatment was achieved in this patient with cyclosporin A (CsA) administration, despite a deficient response to monotherapy with prednisolone (PSL). In addition, we review the previous literature on PLGE associated with MCTD.

Case Report

A 44-year-old Japanese woman with hypoalbuminemia and Raynaud's phenomenon, finger stiffness, edema in the lower legs, and abdominal distension that had persisted for 1 year was admitted to our hospital. She had received albumin supplementation therapy at another hospital because she had experienced facial and conjunctival edema simultaneously with malaise and a periodic fever two months prior to admission. A physical examination revealed a body temperature of $37.3^{\circ} \mathrm{C}$, submandibular and cervical lymphadenopathies, and swollen fingers with slight skin thickness on the distal portions and edematous findings on her face, bulbar conjunctiva, and lower legs.

A laboratory examination revealed decreased serum levels of total protein and albumin (4.1 and $1.3 \mathrm{~g} / \mathrm{dL}$, respectively), although indicators of the hepatic and renal function were within the normal range, and there was no significant protein leakage in the urine. An increase in the erythrocyte sedimentation rate $(105 \mathrm{~mm} / \mathrm{h}$; normal, $<10 \mathrm{~mm} / \mathrm{h})$ was

Department of Medicine (Neurology \& Rheumatology), Shinshu University School of Medicine, Japan 


\section{2 hours}
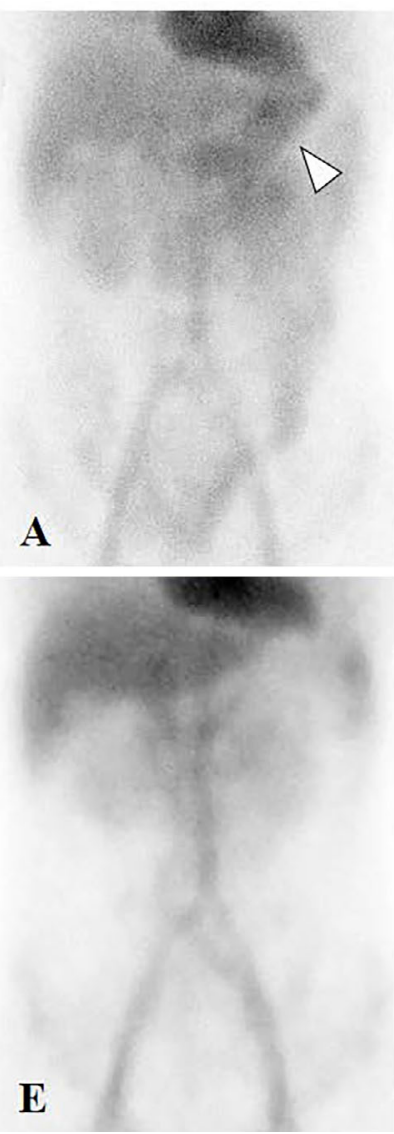

4 hours

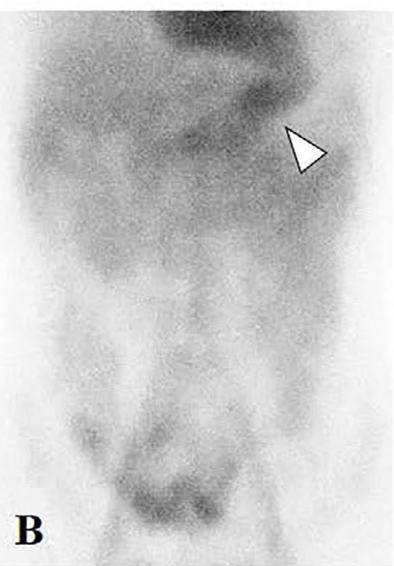

F
6 hours

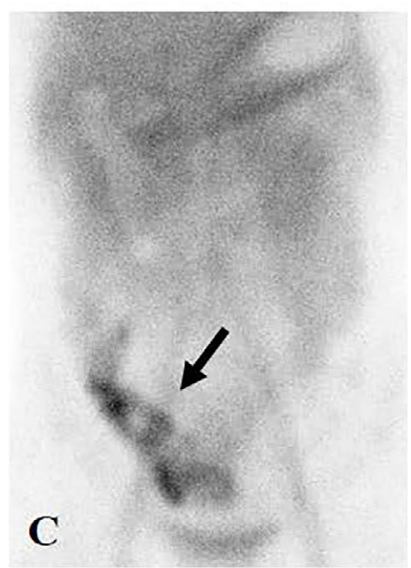

G
24 hours

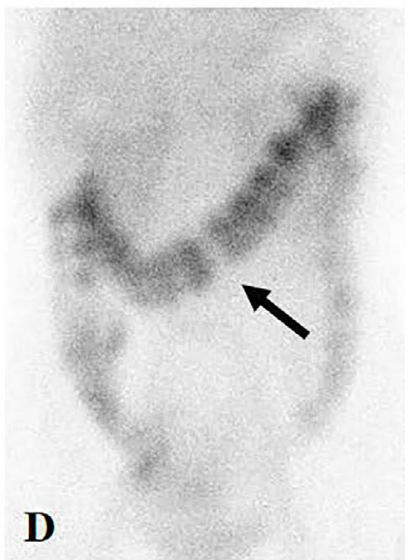

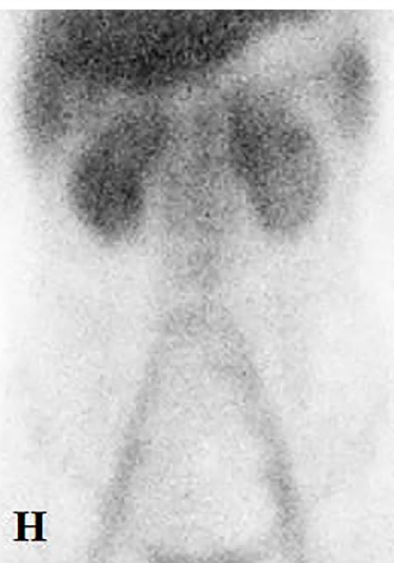

Figure 1. ${ }^{99 m}$ Tc-labeled human serum albumin scintigraphy showing 2-hourly images of albumin leakage prior to treatment (A-D) and after treatment (E-H). Radioactivity accumulation was detected in the stomach (white triangle) at 2 and 4 hours (A, B) and in the intestine (black arrow) at 6 and 24 hours $(C, D)$ after injection of the radioisotope. No abnormal radioactivity was detected at any time point.

shown, but the $\mathrm{C}$-reactive protein levels were within the normal range $(0.05 \mathrm{mg} / \mathrm{dL}$; normal, $<0.10 \mathrm{mg} / \mathrm{dL})$. In addition, leukocytopenia $(1,450 / \mu \mathrm{L})$, lymphopenia $(380 / \mu \mathrm{L})$, and positive reactions to autoantibodies, namely anti-nuclear antibody (1:2,560, speckled pattern) and anti-U1-RNP antibody (550 U/mL; normal, $<10 \mathrm{U} / \mathrm{mL}$ ), were reported along with low levels of complement factors such as $\mathrm{C}_{3}(49.0 \mathrm{mg} / \mathrm{dL}$; normal, $86-160 \mathrm{mg} / \mathrm{dL})$ and $\mathrm{C}_{4}(14.9 \mathrm{mg} / \mathrm{dL}$; normal, 17-45 $\mathrm{mg} / \mathrm{dL})$, and $\mathrm{CH}_{50}(29.0 \mathrm{U} / \mathrm{mL}$; normal, 30-53 U/mL). However, immune complex (C1q) was undetected in the serum. No positivity for other autoantibodies, including antineutrophil cytoplasmic antibodies specific for either myeloperoxidase (MPO-ANCA) or proteinase-3 (PR3-ANCA), anti-dsDNA, anti-Sm, anti-SS-A, anti-SS-B, anti-Scl-70, anti-centromere antibodies, and anti-RNA polymerase III antibody, was detected. Since the clinical and laboratory findings satisfied the diagnostic criteria proposed by the Japanese Ministry of Health and Welfare (4), the patient was diagnosed with MCTD. No malignancy, infection, or pulmonary hypertension was detected on a systemic assessment at admission, although computed tomography indicated bilat- eral interstitial fibrotic changes in the lower lung fields and ascites.

To clarify the cause of hypoalbuminemia, the gastrointestinal tract was examined. ${ }^{99 \mathrm{~m}} \mathrm{Tc}-$ labeled human serum albumin scintigraphy $\left({ }^{99 \mathrm{~m}} \mathrm{Tc}-\mathrm{HAS}\right)$ showed accumulation of the radioisotope in the stomach after 2 and 4 hours and in the intestine after 6 and 24 hours (Fig. 1). On the basis of these findings, PLGE was diagnosed. Alpha-1-antitrypsin clearance $(\alpha 1-\mathrm{AT})$ could not be examined because of persistent constipation. An endoscopic examination revealed edematous findings in the gastric mucosa (Fig. 2) but no significant findings in the intestinal tract. A histopathological examination of the gastric wall tissue indicated infiltration of lymphocytes and plasma cells without lymphangiectasis or immune complex deposition (Fig. 3).

The hypoalbuminemia persisted even after PSL was administered at a dose of $50 \mathrm{mg}$ daily, suggesting that PSL alone was incapable of suppressing the disease activity (Fig. 4). As a supplementary treatment, azathioprine (AZA) was administered; however, it was terminated because of skin eruptions and thrombocytopenia attributed to the use of 

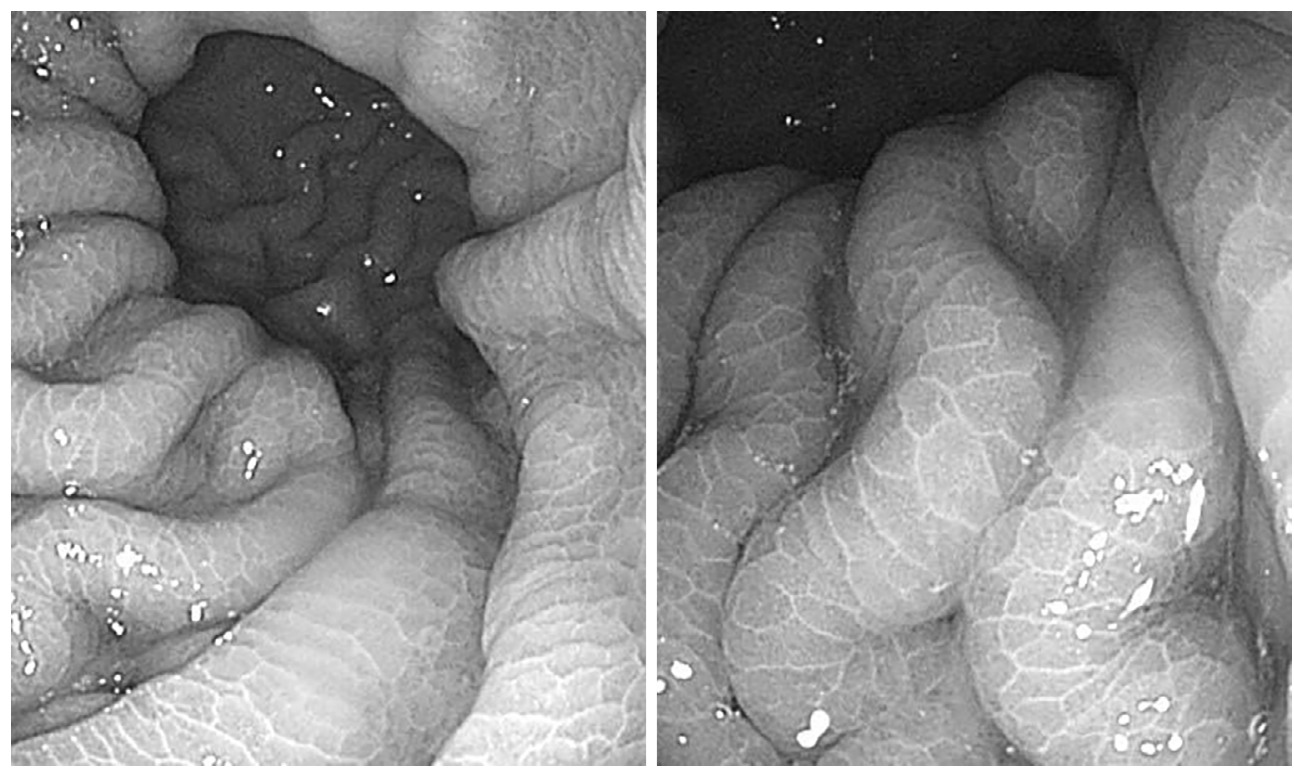

Figure 2. An endoscopic examination showing edematous changes in the gastric mucosa.

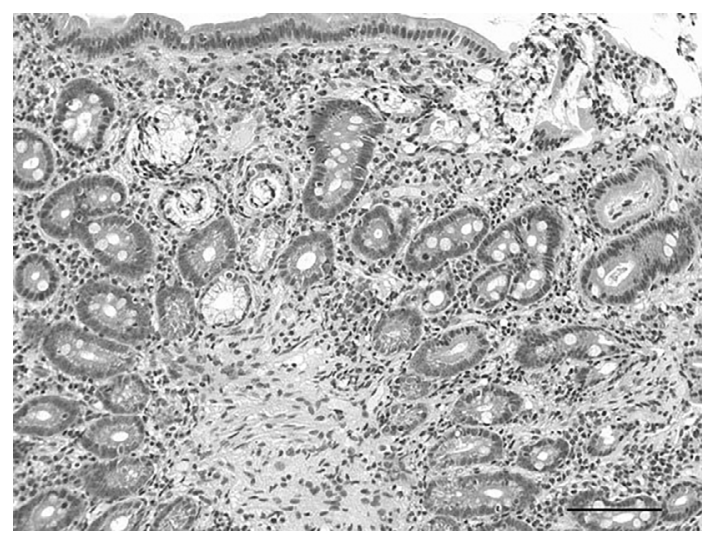

Figure 3. A biopsy specimen from the stomach showing lymphocytes and plasma cells infiltrating mainly the gastric lamina propria (Hematoxylin and Eosin staining, scale bar $=100 \mu \mathrm{m}$ ).

the drug. After recovery from this adverse event, the patient was administered CsA at a dose that ensured its blood trough concentration was between 100 and $150 \mathrm{ng} / \mathrm{mL}$, together with methylprednisolone pulse therapy ( $1 \mathrm{~g}$ daily for 3 days). With this treatment, the albumin leakage detected by ${ }^{99 m}$ Tc-HAS disappeared (Fig. 1), and the serum albumin level returned to the normal range. The patient has shown sustained remission with a constant dose of CsA and a gradually decreasing dose of PSL.

\section{Discussion}

The present case showed leukocytopenia and lymphadenopathy as SLE-like findings along with sclerodactyly and pulmonary fibrotic changes as systemic sclerosis (SSc)-like findings, but general edema and abdominal distension ascribable to albumin leakage were eventually the principal symptoms determining the diagnosis of MCTD in this patient. SLE is a relatively common cause of PLGE associated with autoimmune disease, and the prevalence of PLGE with SLE has been reported to range from $0.94 \%$ to $7.5 \%(2,5,6)$. SSc is also recognized as a cause of PLGE $(7,8)$. In contrast, only seven reports of PLGE associated with MCTD have been described previously, of which two are in Japanese (3, 9-14) (Table).

Of these seven patients, whose cases have been summarized in Table, four concomitantly demonstrated symptoms associated with PLGE when diagnosed with MCTD, suggesting that PLGE can manifest as the initial episode of MCTD. All patients showed peripheral edema, and six also had ascites and pleural and/or pericardial effusion. Abdominal symptoms such as pain or distension were only found in three of the patients. Thus, it appears that regardless of abdominal symptoms, PLGE should be suspected in MCTD unless other causes of hypoalbuminemia, such as hepatic dysfunction and nephritis, are detected.

To establish the diagnosis of PLGE and monitor the efficacy of treatment, $\alpha 1-\mathrm{AT}$ and ${ }^{99 \mathrm{~m}} \mathrm{Tc}-\mathrm{HAS}$ are commonly used. The latter is especially useful for detecting the location of albumin leakage $(2,6,15)$. On reviewing PLGE related to SLE, the small intestine seems to be the most common site of albumin leakage, while the stomach is a rare site, with a leakage frequency of up to $9 \%$, as determined using ${ }^{99 m}$ Tc-HAS $(2,5)$. Interestingly, the stomach was involved as the target organ in the present case and in another case (14), although albumin leakage into the intestine has been reported in many previous patients with MCTD who underwent ${ }^{99 \mathrm{~m}} \mathrm{Tc}-\mathrm{HAS}$ (12-14). In the present patient, an endoscopic examination of the gastrointestinal tract showed no significant visible impairment, such as inflammatory colitis. Furthermore, the significant findings on ${ }^{99 \mathrm{~m}} \mathrm{Tc}-\mathrm{HAS}$ led to the decision regarding the site to biopsy for the histopa- 


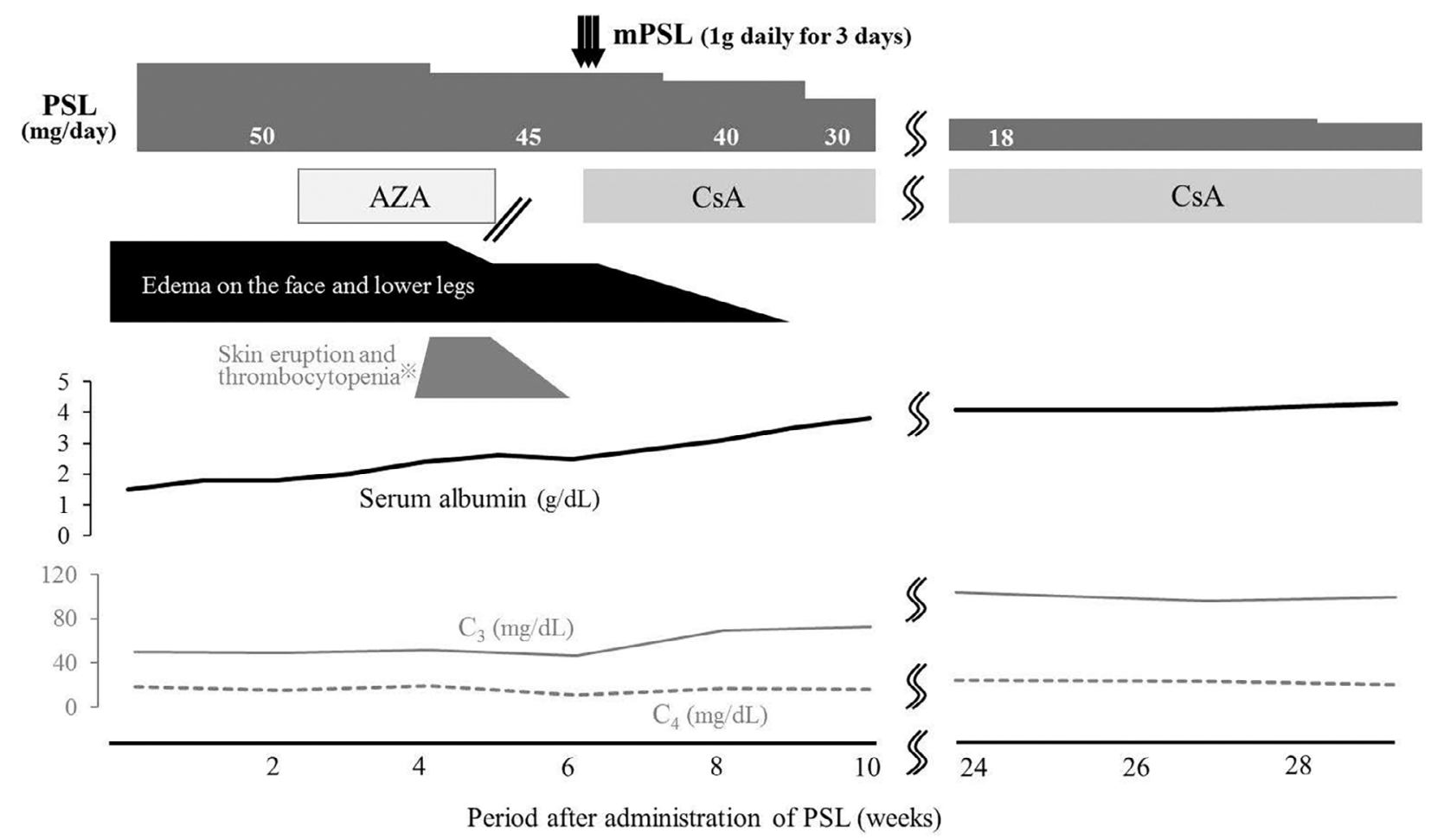

Figure 4. The clinical course of the patient after administration of prednisolone (PSL). *Skin eruptions and thrombocytopenia ascribable to the adverse event of azathioprine (AZA). mPSL: methylprednisolone pulse therapy, CsA: cyclosporin A

thological investigation.

With regard to the histopathological analysis of PLGE related to MCTD, four patients including the present one showed lymphocytic and/or plasmocytic infiltration, three had mucosal edema, two had lymphangiectasis, and one had immune complex deposition (Table). PLGE related to autoimmune diseases, including SLE and SSc, has been proposed to occur because of mucosal capillary permeability derived by complement-mediated or cytokine-mediated vascular damage, non-necrotizing vasculitis, and mucosal lacteal due to lymphangiectasis $(3,6-8,16)$; however, the pathomechanism of PLGE related to MCTD remains unclear. A previous study found that the most frequent finding in PLGE associated with SLE was inflammatory cell infiltration $(73 \%)$; normal cellular morphology had a frequent of $20 \%$, and lymphangiectasis and vasculitis had frequencies of $15 \%$ and $2 \%$, respectively (2). PLGE related to SSc may not be identical, as histological findings both with and without lymphangiectasis were demonstrated, and the latter in particular was accompanied by immune complex deposition in the lamina propria $(7,8)$. Considering these findings, a tissue biopsy may not be entirely definitive for explaining the pathogenesis of PLGE in not only MCTD but also SLE and SSc.

In the present case, additional immunosuppressant therapy was required to achieve a clinical response because monotherapy with PSL was insufficient to suppress the disease activity. In previous cases, three patients required intrave- nous cyclophosphamide (IVCY) together with PSL; furthermore, two patients who received PSL alone experienced relapse (Table). With regard to PLGE in SLE, more than $60 \%$ of patients eventually required additional immunosuppressants, including AZA, IVCY, and methotrexate, as part of combination therapy with PSL to maintain clinical remission $(2,5,6)$. Accordingly, co-administration of an immunosuppressant with PSL may be necessary in order to achieve favorable outcomes in PLGE related to connective tissue diseases.

However, the use of CsA has been reported only in a few cases of PLGE related to $\operatorname{SLE}(16,17)$ and in only one case of PLGE related to CREST syndrome (8). Both early and reliable efficacy by immunosuppressants were required in the present case, as the therapeutic effect was still insufficient even after recovery from the adverse events incurred due to AZA. One reported advantage of CsA is the early appearance of a therapeutic effect compared with other immunosuppressive agents (18-20), and CsA was found to be effective in a case of refractory gastrointestinal involvement with MCTD (21). The present patient was treated with PSL and CsA, which ultimately ensured clinical remission and brought about PSL-sparing effects, suggesting that CsA is a useful agent for treating intractable PLGE associated with MCTD.

In conclusion, PLGE is a rare complication of MCTD, and its pathogenesis remains unclear. Since few cases have been reported so far, it is necessary to accumulate further 


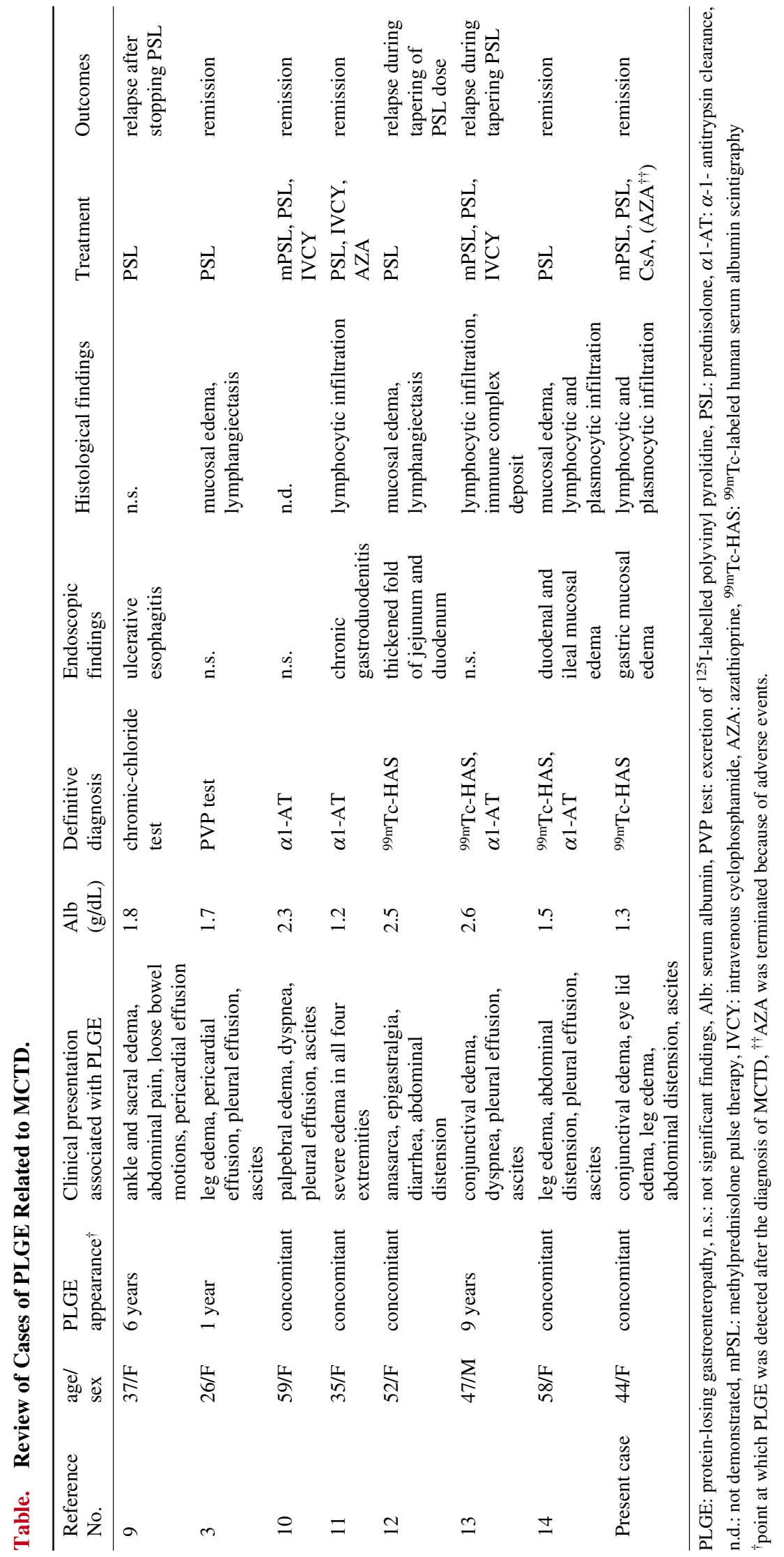

clinical experience regarding PLGE to clarify the details of this disease.

\section{Patient consent}

Informed consent was obtained from this patient.

The authors state that they have no Conflict of Interest (COI). 


\section{References}

1. Umar SB, DiBaise JK. Protein-losing enteropathy: case illustrations and clinical review. Am J Gastroenterol 105: 43-49, 2010.

2. Al-Mogairen SM. Lupus protein-losing enteropathy (Luple): a systematic review. Rheumatol Int 31: 995-1001, 2011.

3. Tsutsumi A, Sugiyama T, Matsumura R, et al. Protein losing enteropathy associated with collagen diseases. Ann Rheum Dis 50: 178-181, 1991.

4. Kasukawa R. Mixed connective tissue disease. Intern Med 38: 386-393, 1999.

5. Mok CC, Ying KY, Mak A, To $\mathrm{CH}$, Szeto ML. Outcome of protein-losing gastroenteropathy in systemic lupus erythematosus treated with prednisolone and azathioprine. Rheumatology (Oxford) 45: 425-429, 2006.

6. Chen Z, Li MT, Xu D, et al. Protein-losing enteropathy in systemic lupus erythematosus: 12 years experience from a Chinese Academic Center. PLoS One 9: e114684, 2014.

7. van Tilburg AJ, van Blankenstein M, Verschoor L. Intestinal lymphangiectasia in systemic sclerosis. Am J Gastroenterol 83: 14181419, 1988.

8. Sunagawa T, Kinjo F, Gakiya I, et al. Successful long-term treatment with cyclosporin A in protein losing gastroenteropathy. Intern Med 43: 397-399, 2004.

9. Terren P. Protein-losing enteropathy and mixed connective-tissue disease. Med J Aust 149: 558-559, 1988.

10. Furuya T, Suzuki T, Onoda N, et al. Mixed connective tissue disease associated with protein losing enteropathy: successful treatment with intravenous cyclophosphamide therapy. Intern Med 31: 1359-1362, 1992.

11. Chao CT, Lai CY, Chang PL, Wu HS, Wu VC, Wang WJ. Mixed connective tissue disease with protein-losing enteropathy: discussion of the treatment strategy. J Clin Rheumatol 17: 286-287, 2011.

12. Kashihara T, Fujimori E, Oki A, et al. Protein-losing enteropathy and pancreatic involvement in a case of connective tissue disease.
Gastroenterol Jpn 27: 246-251, 1992.

13. Nosho K, Takahashi H, Ikeda $Y$, et al. A case of protein-losing gastroenteropathy in association with mixed connective tissue disease which was successfully treated with cyclophosphamide pulse therapy. Ryumachi 38: 818-824, 1998 (in Japanese).

14. Kakigao K, Fukushima N, Mizutani T, et al. A case of proteinlosing gastroenteropathy accompanied by Sjogren syndrome and mixed connective tissue disease. Nihon Shokakibyo Gakkai Zasshi 109: 1770-1775, 2012 (in Japanese).

15. Chiu NT, Lee BF, Hwang SJ, Chang JM, Liu GC, Yu HS. Proteinlosing enteropathy: diagnosis with ${ }^{99 \mathrm{~m}} \mathrm{Tc}$-labeled human serum albumin scintigraphy. Radiology 219: 86-90, 2001.

16. Law ST, Ma KM, Li KK. The clinical characteristics of lupus related protein-losing enteropathy in hong kong chinese population: 10 years of experience from a regional hospital. Lupus 21: 840847, 2012.

17. Chang YS, Lai CC, Chen WS, Wang SH, Chou CT, Tsai CY. Protein-losing enteropathy and premature ovarian failure in a young woman with systemic lupus erythematosus. Lupus 21: 1237-1239, 2012.

18. Ejstrup L. Severe dermatomyositis treated with cyclosporin A. Ann Rheum Dis 45: 612-613, 1986.

19. Shimojima $\mathrm{Y}$, Ishii $\mathrm{W}$, Kato $\mathrm{T}$, et al. Intractable skin necrosis and interstitial pneumonia in amyopathic dermatomyositis, successfully treated with cyclosporin A. Intern Med 42: 1253-1258, 2003.

20. Correia O, Polonia J, Nunes JP, Resende C, Delgado L. Severe acute form of adult dermatomyositis treated with cyclosporine. Int J Dermatol 31: 517-519, 1992.

21. Maeda N, Abe S, Yoshizawa T, et al. Low-dose cyclosporin for multiple colonic ulcers associated with mixed connective tissue disease. Clin Rheumatol 18: 410-413, 1999.

The Internal Medicine is an Open Access article distributed under the Creative Commons Attribution-NonCommercial-NoDerivatives 4.0 International License. To view the details of this license, please visit (https://creativecommons.org/licenses/ by-nc-nd/4.0/).

(C) 2017 The Japanese Society of Internal Medicine http://www.naika.or.jp/imonline/index.html 